\title{
Toward A Measurement Model of Fuzzy Prioritization Operators
}

\author{
Kevin Kam Fung Yuen \\ Department of Industrial and Systems Engineering, The Hong Kong Polytechnic Univer- \\ sity, Hung Hom, Kowloon, Hong Kong \\ kevinkf.yuen@gmail.com, ise.kevinyuen@polyu.edu.hk
}

\begin{abstract}
The Fuzzy Prioritization Operators (FPOs) have been studied by various studies. As various FPOs produce different results, the fitness levels of FPOs are necessary to be measured. This research reviews two important FPOs, and proposes a Fuzzy Prioritization Measurement (FPM) model to measure the appropriateness of them. The advantage of FPM is to enhance the decision quality of the fuzzy AHP by choosing the best FPO with the most fitness.
\end{abstract}

Keywords: Fuzzy AHP, Fuzzy Prioritization Operator, Fuzzy Optimization, Fuzzy Decision Analysis

\section{Introduction}

Applications of the Analytic Hierarchy Process (AHP) and the Fuzzy Analytic Hierarchy Process increasingly address the attentions of the industry applications and scholar research.

The Analytic Hierarchy Process [4] is the popular model to aggregate multiple criteria for decision making. The limitation is that the measurement scale for the value of the utility function, which is basically numerical and probabilistically judgmental, induces evaluation problem. This introduces the studies of fuzzy AHP [e.g. 1-3,5-7,9,12-13] to address the limitation.

The Extent Analysis Method (EAM) on fuzzy AHP [2] has been used in many studies as it is regarded as less complexity. However, Wang at el. [7] pointed out this method was problematic. $[6,7]$ proposed modified fuzzy Logarithmic Least Squares Method (LLSM) as the appropriate alternative on the basis of $[1,5]$. In order to fairly criticize both methods, this study only focuses on the discussion of the prioritization process in both methods, and proposes the Fuzzy Prioritization Measurement (FPM) Model to measure their fitness levels for a fuzzy comparison matrix.

The structure of this article is as follows: Section 2 introduces the fundamental concepts of AHP and Fuzzy AHP. Section 3 introduces the two importance prioritization operators in Fuzzy AHP. The Fuzzy Prioritization Measurement model is proposed in section 4. Two numerical examples are illustrated in section 5. Section 6 concludes the contribution of this work and proposes the extensions of this research.

\section{Preliminary Knowledge}

\subsection{Analytic Hierarchy Process}

The AHP includes three core processes: assessment, prioritization, and synthesis. In the assessment, verbal judgments are given by decision makers for pairwise comparisons. The verbal judgment is usually on a 9 point verbal scale represented by crisp number: 1 for equal im-

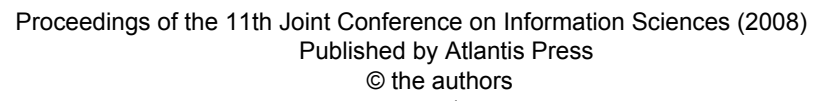


portance, 2 for weak importance, and finally 9 for extreme importance. For pairwise comparisons, $a_{i j}$ is a numeric point to estimate the relative importance of object i over object $\mathrm{j}$, and $A=\left\{a_{i j}\right\}$, $0<a_{i j}=a_{j i}^{-1}, i, j=1,2, \ldots, n$ is a pairwise comparison matrix. Thus a pairwise comparison matrix is also called a reciprocal matrix. The reciprocal matrices of all assessments are formed by transforming the linguistic labels to numerical values. In the prioritization process, a local priority vector $W=\left\{w_{1}, \ldots, w_{n}\right\}$, $\sum_{i=1}^{n} w_{i}=1$ is generated from a reciprocal matrix $A$ by a Prioritization Operator (PO), i.e. $P O: A \rightarrow W$. In the synthesis stage, a set of these local priority vectors W's are aggregated as a global priority vector $V=\left\{v_{1}, \ldots, v_{n}\right\}$ by an aggregation operator Agg $:\{W\} \rightarrow V$.

These processes are with three fundamental problems: (i) selection of numerical scales in stage one; (ii) selection of prioritization operators (or methods) in stage two; (iii) selection of the aggregation operators. Problem (i) is addressed by [8; 13] whilst problem (ii) is addressed by [9]. And Problem (iii) can be referred to [10].

\subsection{Fuzzy Analytic Hierarchy Process}

The Fuzzy AHP comprises of two types of core processes. Type I includes fuzzy assessment, fuzzy prioritization, defuzzification, and crisp synthesis. Type II includes: fuzzy assessment, fuzzy prioritization, and fuzzy synthesis. Extend Analysis Method [2] is Type I whilst modified Fuzzy LLSM $[6,7]$ is Type II.

In the Fuzzy Assessment, a fuzzy comparison matrix is expressed by

$$
\begin{aligned}
& \bar{A}=\left(\bar{a}_{i j}\right)_{n \times n}= \\
& \left(\begin{array}{cccc}
(1,1,1) & \left(l_{12}, m_{12}, u_{12}\right) & \ldots & \left(l_{1 n}, m_{1 n}, u_{1 n}\right) \\
\left(l_{21}, m_{21}, u_{21}\right) & (1,1,1) & \cdots & \left(l_{2 n}, m_{2 n}, u_{2 n}\right) \\
\vdots & \vdots & \ddots & \vdots \\
\left(l_{n 1}, m_{n 1}, u_{n 1}\right) & \left(l_{n 2}, m_{n 2}, u_{n 2}\right) & \cdots & (1,1,1)
\end{array}\right)
\end{aligned}
$$

,where

$\bar{a}_{i j}=\left(l_{i j}, m_{i j}, u_{i j}\right)=\bar{a}_{j i}^{-1}=\left(1 / u_{j i}, 1 / m_{j i}, 1 / l_{j i}\right)$ ,for $i, j=1, \ldots, n$ and $i \neq j . \quad \bar{a}_{i j}=(1,1,1)$ if $i=j$.

The verbal judgment is usually on a 9 point verbal scale represented by fuzzy numbers: $(1,1,1)$ for equal importance, $(1.5,2,2.5)$ for weak importance, and finally $(8.5,9,9.5)$ for extreme importance. In the fuzzy prioritization, $\bar{A}$ is derived as a vector of fuzzy priorities or fuzzy relative weights $\tilde{W}=\left\{\tilde{w}_{i}\right\}$ and $\tilde{w}_{i}=\left(w_{i}^{U}, w_{i}^{M}, w_{i}^{L}\right)$. These two steps are the same in the type I and type II methods, but following steps are different.

In type I method, each $\tilde{w}_{i}$ is defuzzified as a crisp number, and then these crisp numbers is synthesized. This synthesized step is the same as the crisp AHP. The aggregation technique Agg: $\{W\} \rightarrow V$ is usually the weight average method. Thus the final value is the crisp number.

In type II method, each $\tilde{w}_{i}$ is directly aggregated as a global fuzzy priority vector $\bar{V}=\left\{\bar{v}_{1}, \ldots, \bar{v}_{n}\right\}, \bar{v}_{i}=\left(\tilde{v}_{i}^{U}, \tilde{v}_{i}^{M}, \tilde{v}_{i}^{L}\right)$ by a fuzzy aggregation operator FAgg $:\{\bar{W}\} \rightarrow \bar{V}$.

The problems of Fuzzy AHP are similar to the generic AHP problems as Fuzzy AHP is the extension of AHP. This research only focuses discussion of the evaluation of FPOs.

\section{Fuzzy Prioritization Operators}

There are various computational models for fuzzy AHP. This paper chooses two 
importance fuzzy prioritization Operators for discussion: Extent Analysis Method and Modified Fuzzy LLSM.

\subsection{Extent Analysis Method}

Chang [2] proposed an Extend Analysis Method to derive the priority of a fuzzy comparison matrix with five steps as follows:

Step 1: sum up each row of $\bar{A}$ by fuzzy addition:

$$
\begin{aligned}
& R S_{i}=\sum_{j=1}^{n} a_{i j}=\left(\sum_{j=1}^{n} l_{i j}, \sum_{j=1}^{n} m_{i j}, \sum_{j=1}^{n} u_{i j}\right), \\
& i=1, \ldots, n
\end{aligned}
$$

Step 2: normalize $R S_{i}, i=1, \ldots, n$ by

$$
\begin{aligned}
S_{i} & =\frac{R S_{i}}{\sum_{j=1}^{n} R S_{j}} \\
& =\left(\frac{\sum_{j=1}^{n} l_{i j}}{\sum_{i=1}^{n} \sum_{j=1}^{n} u_{i j}}, \frac{\sum_{j=1}^{n} m_{i j}}{\sum_{i=1}^{n} \sum_{j=1}^{n} m_{i j}}, \frac{\sum_{j=1}^{n} u_{i j}}{\sum_{i=1}^{n} \sum_{j=1}^{n} l_{i j}}\right) \\
, i & =1, \ldots, n
\end{aligned}
$$

Step 3: computer the degree of possibility of $S_{i} \geq S_{j}$ by

$$
V\left(S_{i} \geq S_{j}\right)=\left\{\begin{array}{lr}
1, & m_{i} \geq m_{j} \\
\frac{u_{i}-l_{j}}{\left(u_{i}-m_{i}\right)+\left(m_{i}-l_{j}\right)}, l_{j} \leq u_{j} \\
0, & \text { otherwise }
\end{array}\right.
$$

$, i, j=1, \ldots, n ; j \neq i, S_{i}=\left(l_{i}, m_{i}, u_{i}\right)$ and

$S_{j}=\left(l_{j}, m_{j}, u_{j}\right)$.

Step 4: calculate the degree of possibility of $S_{j}$ over all the other (n-1) fuzzy numbers by

$$
\begin{aligned}
& V\left(S_{i} \geq S_{j}: j=1, \ldots, n ; j \neq i\right)= \\
& \min _{j \in\{i, \ldots, n\}, j \neq i} V\left(S_{i} \geq S_{j}\right), i=1, \ldots, n
\end{aligned}
$$

Step 5: the priority vector $W=\left(w_{1}, \ldots, w_{n}\right)$ of $\bar{A}$ is the form:

$$
w_{i}=\frac{V\left(S_{i} \geq S_{j}: j=1, \ldots, n ; j \neq i\right)}{\sum_{k=1}^{n} V\left(S_{k} \geq S_{j}: j=1, \ldots, n ; j \neq k\right)}
$$

$, i=1, \ldots, n$

Many fuzzy AHP applications used Chang's model [2]. Wang [7] pointed out some shortcomings of Chang's model and proposed the modified fuzzy LLSM on the basis of previous studies $[1,3]$.

\subsection{Modified Fuzzy LLSM}

The modified fuzzy LLSM [6, 7] derives the priorities of the triangular fuzzy comparison matrix. The FPO of MF-LLSM has following form:

$$
\text { Min } \begin{aligned}
J=\sum_{i=1}^{n} \sum_{j=1, j \neq i}^{n} & \left(\left(\ln w_{i}^{L}-\ln w_{j}^{U}-\ln l_{i j}\right)^{2}\right. \\
+ & \left(\ln w_{i}^{M}-\ln w_{j}^{M}-\ln m_{i j}\right)^{2} \\
+ & \left.\left(\ln w_{i}^{U}-\ln w_{j}^{L}-\ln u_{i j}\right)^{2}\right)
\end{aligned}
$$

Subject to

$$
\left\{\begin{array}{l}
w_{i}^{L}+\sum_{j=1, j \neq i}^{n} w_{j}^{U} \geq 1 \\
w_{i}^{U}+\sum_{j=1, j \neq i}^{n} w_{j}^{L} \leq 1 \\
\sum_{i=1}^{n} w_{i}^{M}=1 \\
\sum_{i=1}^{n}\left(w_{i}^{L}+w_{i}^{U}\right)=2 \\
w_{i}^{U} \geq w_{i}^{M} \geq w_{i}^{L}>0
\end{array} \quad i=1, \ldots, n\right.
$$

The optimum solution to the above model forms normalized a vector of triangular 
fuzzy weights $\tilde{w}_{i}=\left(w_{i}^{U}, w_{i}^{M}, w_{i}^{L}\right)$, $i=1, \ldots, n$.

\subsection{Remarks}

It can be observed that EAM produces fuzzy relative weights from the Fuzzy Normalized Row Sum Method (NRSM) from Step 1 to Step 2 in fact. These fuzzy weights are finally converted to a crisp weight value. On the other hand, the MFLLSM produces a fuzzy value by fuzzy optimization method. It is more appropriate to compare MF-LLSM and NRSM of EAM. Thus next section develops a Fuzzy Prioritization Measurement Model to address this comparison issue. The matters of the measurement of defuzzification and aggregation problems are beyond the scope of this paper.

\section{Fuzzy Prioritization Measurement Model}

The Fuzzy Prioritization Measurement Model (FPMM) evaluates the validity of the prioritization operators. Two variance methods in crisp AHP scenarios are reviewed for the development of FPMM. In classical AHP problem, to measure the distribution of the variance, one approach is to use Root Mean Square Variance which has the form [9]:

$$
\operatorname{RMSV}(A, W)=\sqrt{\frac{1}{n \times n} \sum_{i=1}^{n} \sum_{j=1}^{n}\left(a_{i j}-\frac{w_{i}}{w_{j}}\right)^{2}}
$$

A is a pairwise matrix $\left\{a_{i j}\right\}, W$ is a priorities vector of a prioritization operator, and $w_{i}, w_{j} \in W \in\left\{W_{1}, \ldots, W_{K}\right\}=\{W\}$. If $\frac{1}{n \times n}$ is taken out, the new form is Euclidean Distance, which was used by [3]. For easier interpretation of the result, it is more appropriate to use the average of the value. Thus RMSV is preferred.
However, a limitation of RMSV is that the weights for the penalty are not justified. For example, the penalty of the condition

$\left(w_{i}>w_{j} \& a_{i j}<1 \& a_{i j} \neq \frac{w_{i}}{w_{j}}\right)=$ True is not the same as the one of the condition $\left(w_{i}>w_{j} \& a_{i j}>1 \& a_{j i} \neq \frac{w_{j}}{w_{i}}\right)=$ True.

To determine the variance associated with weights, Minimum Violation [3] was proposed as weight determination.

$$
\begin{aligned}
& M V(A, W)=\sum_{i} \sum_{j} I_{i j} \\
& I_{i j}=\left\{\begin{array}{c}
1, w_{i}>w_{j} \quad \& \quad a_{j i}>1 \\
0.5, w_{i}=w_{j} \text { \& } a_{j i} \neq 1 \\
0.5, w_{i} \neq w_{j} \text { \& } a_{j i}=1 \\
0 \quad, \text { Otherwise }
\end{array}\right.
\end{aligned}
$$

However, as the value of $M V$ depends on the size $\left(n^{2}\right)$ of the matrix (usually a larger sized matrix leads to a higher value of $\mathrm{MV}$ ), the mean value of $M V$ is more appropriate for measuring POs. In addition, a mistake of above definition of $I_{i j}$ is that the condition $w_{i}<w_{j} \& a_{j i}<1$ scores 0 . Thus The MMV with correction definition of $I_{i j}$ has the form:

$$
\begin{aligned}
& \operatorname{MMV}(A, W)=\frac{1}{n^{2}}\left(\sum_{i} \sum_{j} I_{i j}\right) \quad \text {,where; } \\
& I_{i j}=\left\{\begin{array}{cccc}
1, w_{i}>w_{j} & \& & a_{j i}>1 \\
1 & w_{i}<w_{j} & \& & a_{j i}<1 \\
0.5, & w_{i}=w_{j} & \& & a_{j i} \neq 1 \\
0.5, w_{i} \neq w_{j} & \& & a_{j i}=1 \\
0 & , \text { Otherwise }
\end{array}\right.
\end{aligned}
$$

Even though the new correction form is applied, another limitation of MMV is that it takes care of the penalty scores only, and ignores the actual variance values. 
To combine the advantages of Root Mean Square Variance and Mean Minimum Violation, as well as offset their shortages, this paper proposes the Weighted Root Mean Square Variance method, which is expressed as:

$$
\begin{aligned}
& \sigma=W R M S V(A,\{W\})=\sqrt{\sum_{i} \sum_{j} \frac{Y_{i j}}{n \times n}}, \\
& Y_{i j}=\left\{\begin{array}{r}
\beta_{1}\left(a_{i j}-\frac{w_{i}}{w_{j}}\right)^{2}, w_{i}>w_{j} \quad \& a_{i j}>1 \\
\text { or } w_{i}<w_{j} \& a_{i j}<1 \\
\text { or } w_{i}=w_{j} \& a_{i j}=1 \\
\beta_{2}\left(a_{i j}-\frac{w_{i}}{w_{j}}\right)^{2}, w_{i}=w_{j} \& a_{i j} \neq 1 \\
\text { or } w_{i} \neq w_{j} \& a_{i j}=1 \\
\beta_{3}\left(a_{i j}-\frac{w_{i}}{w_{j}}\right)^{2}, \text { otherwise }
\end{array}\right.
\end{aligned}
$$

, where $1=\beta_{1} \leq \beta_{2} \leq \beta_{3}$.

$\beta_{1}, \beta_{2}, \beta_{3}$ are the penalty weights. RMSV is the special case of WRMSV if $\beta_{1}=\beta_{2}=\beta_{3}=1$. By default setting of WRMSV, $\quad \beta_{1}=1, \beta_{2}=3, \beta_{3}=10$ are defined.

Next, WRMSV is extended as a Fuzzy WRMSV by considering a modal value and two interval values. Thus FWRMSV is the form:

$$
\begin{aligned}
& \bar{\sigma}=\left(\sigma^{L}, \sigma^{M}, \sigma^{U}\right), \text { where } \\
& \sigma^{L}=W R M S V\left(\{l\},\left\{w_{i}^{L}\right\}\right) \\
& \sigma^{M}=W R M S V\left(\{m\},\left\{w_{i}^{M}\right\}\right) \\
& \sigma^{U}=W R M S V\left(\{l\},\left\{w_{i}^{U}\right\}\right)
\end{aligned}
$$

FPM model is the aggregation of FWRMSV defined as follows:

$$
\begin{aligned}
& \hat{\sigma}=\alpha^{L} \cdot \sigma^{L}+\alpha^{M} \cdot \sigma^{M}+\alpha^{U} \cdot \sigma^{U} \\
& \text {, where } \alpha^{M} \geq \alpha^{L} \text { or } \alpha^{M} \geq \alpha^{U} \quad \text {, and } \\
& \alpha^{L}+\alpha^{M}+\alpha^{U}=1 .
\end{aligned}
$$

By default $\quad \alpha^{M}=0.5 \quad$ and $\alpha^{L}=\alpha^{U}=0.25$.

\section{Numerical Examples}

Two examples are illustrated: one is from [7], and another one is proposed by this paper.

Consider two decision criteria with their fuzzy relative weights [7]: $\tilde{w}_{1}=(0.65$, $0.7,0.75)$ and $\tilde{w}_{2}=(0.25,0.3,0.35)$. Thus the fuzzy comparison matrix is

$$
\bar{A}=\left[\begin{array}{cc}
(1,1,1) & (1.8571,2.333,3) \\
(0.3333,0.4286,0.5385) & (1,1,1)
\end{array}\right]
$$

By using Fuzzy Normalized Row Sum Method (FNRS) of EAM, $\tilde{w}_{1}=(0.516$, $0.700,0.955)$, and $\tilde{w}_{2}=(0.24,0.3,0.367)$. For the FWRMSV $\bar{\sigma}=(0.158,0,0.2143)$. The aggregation of FWRMSV $\hat{\sigma}$ is 0.093 .

By using modified fuzzy LLSM, $\tilde{w}_{1}=(0.65, \quad 0.700, \quad 0.75), \quad$ and $\tilde{w}_{2}=(0.250,0.300,0.350)$. Then $\bar{\sigma}=$ (0.372, 0, 0.430), and $\hat{\sigma}=0.201$, which is larger than FNRS.

Another example is that

$$
\bar{A}=\left[\begin{array}{ccc}
(1,1,1) & (1,2,3) & (3,4,5) \\
(1 / 3,1 / 2,1) & (1,1,1) & (1,2,3) \\
(1 / 5,1 / 4,1 / 3) & (1 / 3,1 / 2,1) & (1,1,1)
\end{array}\right] .
$$

For FNRS, $\bar{\sigma}=(0.741,0,0.740)$, and $\hat{\sigma}=0.370$. For modified fuzzy LLSM, $\bar{\sigma}=(0.847,0,0.788)$, and $\hat{\sigma}=0.409$, which is also larger than FNRS.

These can be concluded that although [7] proved EAM may produce wrong decision. However, in this study, modified fuzzy LLSM produces higher aggregated value of FWRMSV than FNRS of EAM does if the fuzzy prioritization process is considered only. This means that the 
modified fuzzy LLSM may produce rank reversals due to the approximated fitness is lower than FNRS. Future study investigates this issue in depth.

\section{Conclusion and Future Study}

This paper proposes a Fuzzy Prioritization Measurement model to measure the appropriateness of the fuzzy prioritization operators. The contribution of this research is that the foundation to evaluate the fuzzy prioritization operators is established for several directions of the future studies. The extensions of this study will investigate more FPOs, propose some new fuzzy prioritization operators with less FWRMSV, and perform the comprehensive numerical analysis of numerous fuzzy matrices.

\section{Acknowledgements}

The author wishes to thank the Research Office of the Hong Kong Polytechnic University for support of this research project.

\section{References}

[1] C.G.E. Boender, J.G. de Graan, F.A. Lootsma, Multi-criteria decision analysis with fuzzy pairwise comparisons, Fuzzy Sets and Systems 29 (1989) 133- 143

[2] D.Y. Chang, Applications of extend analysis method on fuzzy AHP, European J. Oper. Res. 95 (1996) 649655.

[3] B. Golany, M. Kress, A multicriteria evaluation of methods for obtaining weights from ratio-scale matrices, Eur J Opl Res 69 (1993) 210-220.

[4] T. L. Saaty, The Analytic Hierarchy Process. New York: McGraw-Hill, 1980

[5] P.J.M. van Laarhoven, W. Pedrycz, A fuzzy extension of Saaty's Priority theory, Fuzzy Sets and Systems 11 (1983) 229-24

[6] Y. M. Wang, T. M.S. Elhag, Z. Hua, A modified fuzzy logarithmic least squares method of fuzzy analytic hierarchy process, Fuzzy Sets and Systems 157 (2006) 3055-3071

[7] Y. M. Wang, Y. Luo, Z. Hua, On the extent analysis method for fuzzy AHP and its application, European Journal of Operational Research 186 (2008) 735-747

[8] K.K.F. Yuen, A Compound Linguistic Ordinal Scales Model: A Breakthrough of the Magical Number Seven, Plus or Minus Two. Information Sciences, 2008, under revision.

[9] K.K.F Yuen, Analytic Hierarchy Prioritization Process in the AHP Applications Development: A Prioritization Operator Selection Approach, Applied Soft Computing, 2008, under review.

[10] K.K.F. Yuen, Selection of Aggregation Operators with Decision Attitudes, WSC 2008 Online World Conference on Soft Computing in Industrial Applications, 2008, to appear.

[11] K.K.F. Yuen, H.C.W. Lau, Software Vendor Selection using Fuzzy Analytic Hierarchy Process with ISO/IEC 9126, IAENG International Journal of Computer Science , 2008, to appear.

[12] K.K.F Yuen and H.C.W. Lau, Measuring Software Quality using a Fuzzy Group Analytical Hierarchy Process Model with ISO/IEC 9126, Journal of Computer Science and Technology, 2008, to appear.

[13] K.K.F. Yuen, H.C.W. Lau, A Linguistic Possibility-Probability Aggregation Model for Decision Analysis with Imperfect Knowledge, Applied Soft Computing, 2008, doi:10.1016/j.asoc.2008.08.003

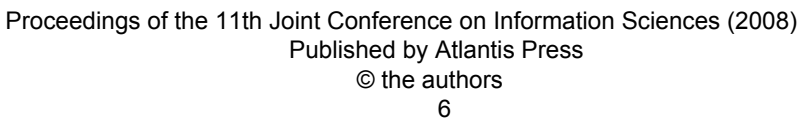

